\title{
Investigation of Electromagnetic Influence on the Liquid Heart of Aluminium Ingots at Continuous Casting in a Direct Chill Mold
}

\author{
Mikhail V. Pervukhin, \\ Mikhail Y. Kuchinskii* and Sergei P. Timofeev \\ Siberian Federal University \\ 79 Svobodny, Krasnoyarsk, 660041, Russia
}

Received 31.05.2018, received in revised form 02.11.2018, accepted 14.11.2019

The mathematical model of system "inductor-ingot" for investigation electromagnetic process at the continuous casting of aluminum ingots in a direct chill mold is considered in this article. Calculation at various parameters of a power line is made, electromechanical characteristics of stirrer are found. The frequency range at which electromagnetic influence of an inductor on a liquid heart of an ingot is most effective is established. The results of mathematical and physical modeling which have shown reliability of mathematical model are compared.

Keywords: electromagnetic stirring, aluminum alloys, mathematical modeling, direct chill mold, continuous casting.

Citation: Pervukhin M.V., Kuchinskii M.Y., Timofeev S.P. Investigation of electromagnetic influence on the liquid heart of aluminium ingots at continuous casting in a direct chill mold, J. Sib. Fed. Univ. Eng. technol., 2019, 12(8), 952-961. DOI: $10.17516 / 1999-494 X-0195$.

\section{Исследование электромагнитного воздействия}

на жидкую сердцевину алюминиевых слитков

при непрерывном литье в кристаллизатор скольжения

\section{М.В. Первухин, М.Ю. Кучинский, С.П. Тимофеев \\ Сибирский федеральный университет Россия, 660041, Красноярск, пр. Свободныий, 79}

В статье рассматривается математическая модель для исследования электромагнитных прочессов в системе «индуктор-слиток» при непрерывном литье алюминиевых слитков в кристаллизатор скольжения. Произведен расчет при различных параметрах питающей сети,

(C) Siberian Federal University. All rights reserved

This work is licensed under a Creative Commons Attribution-NonCommercial 4.0 International License (CC BY-NC 4.0).

* Corresponding author E-mail address: km192@mail.ru 
получены электромеханические характеристики перемешивателя. Установлен диапазон частот, при котором электромагнитное воздействие индуктора на жидкую сердиевину слитка наиболее эффективно. Сопоставлены результаты математического и физического моделирования, показавшие достоверность математической модели.

Ключевые слова: электромагнитное перемешивание, алюминиевые сплавы, математическое моделирование, кристаллизатор скольжения, непрерывное литье.

Специальные алюминиевые сплавы, обладающие высокими технологическими свойствами, имеют важное значение в промышленности. При непрерывном литье алюминиевых слитков для достижения требуемых физико-механических свойств и химического состава требуется перемешивание жидкой фазы с целью равномерного распределения легирующих компонентов. В настоящее время помимо электромагнитного перемешивания известны такие способы физического воздействия на расплавленный металл, как механическое, ультразвуковое, вибрационное. Однако электромагнитное перемешивание является наиболее надежным, так как отсутствует непосредственный контакт между индуктором и слитком, также оно обладает комплексным воздействием на жидкий металл, позволяя регулировать движение расплава в широком диапазоне скоростей. Перемешивание жидкого металла под воздействием электромагнитного поля дает возможность повысить качество слитка за счет получения однородной кристаллической структуры сплава и равномерного химического состава по объему слитка [1].

В настоящее время электромагнитное перемешивание жидкой сердцевины используется в основном на литейных комплексах, предназначенных для получения одиночных крупногабаритных слитков. Установка электромагнитных перемешивателей на многоручьевые литейные комплексы имеет не меньшую актуальность, но затрудняется ограниченным пространством между кристаллизаторами литейного стола. Разработка компактной конструкции электромагнитного перемешивателя для установки на многоручьевые литейные комплексы является актуальной задачей.

На рис. $1 a$ изображен эскиз системы «индуктор-слиток». В кристаллизатор 1 непрерывно поступает жидкий металл 2. Индуктор 3 оказывает силовое воздействие на расплав в результате наведения вихревых токов. Жидкая фаза перемешивается электромагнитными силами, создающими вращающееся электромагнитное поле. Под действием охлаждающей воды, поступающей на боковую поверхность слитка из охладителя 4, жидкая масса непрерывно затвердевает, превращаясь в слиток. Граница раздела твердой и жидкой фаз образует фронт кристаллизации 5. Твердая зона слитка 6 вытягивается вниз $[2,3]$.

На рис. 16 представлен эскиз электромагнитного перемешивателя, состоящего из катушек 1 и магнитопровода 2. В качестве материала магнитопровода используют листы электротехнической стали, собранные в пакеты с пазами со стороны слитка. Конструкция включает в себя шесть катушек, которые располагаются на незначительном расстоянии от слитка для достижения максимального КПД установки. Корпус кристаллизатора выполнен из алюминиевого сплава.

Электрическая схема подключения катушек приведена на рис. 2. Катушки подключены к трехфазной питающей сети, сила тока в проводнике I $=5 \mathrm{~A}$, что обусловлено использованием

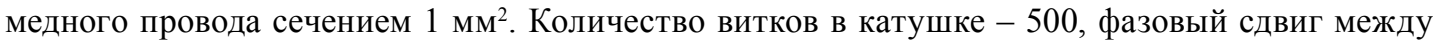
токами обмоток составляет $60^{\circ}$.

$$
-953-
$$



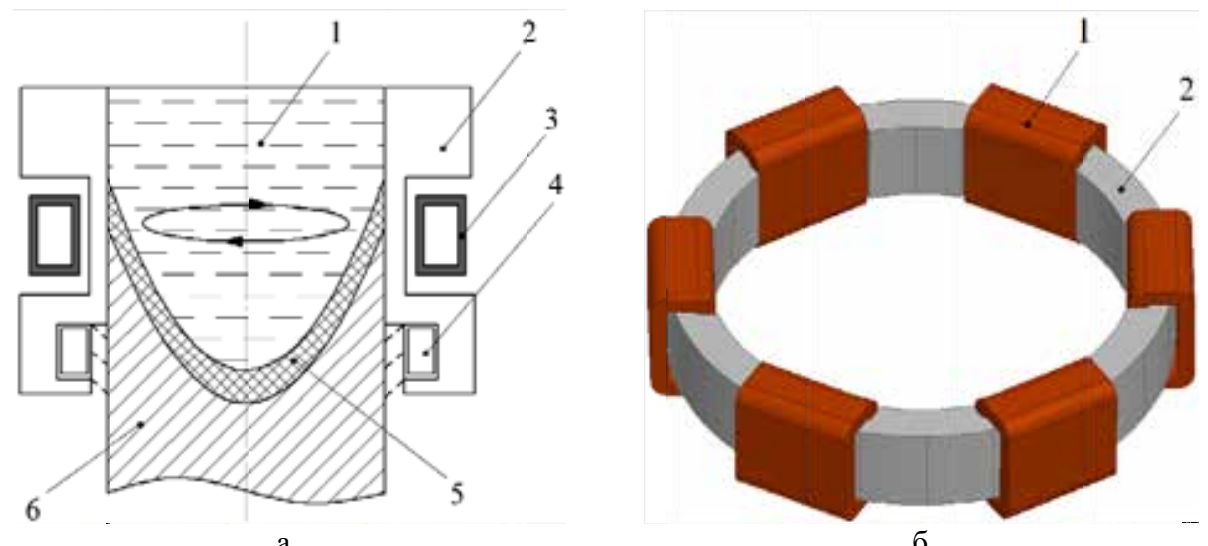

6

Рис. 1. Схема перемешивания жидкой сердцевины электромагнитным полем (а) и общий вид конструкции электромагнитного перемешивателя (б)

Fig. 1. The stirring scheme of the liquid heart with an electromagnetic field (a) and general view of the design of the electromagnetic stirrer (б)

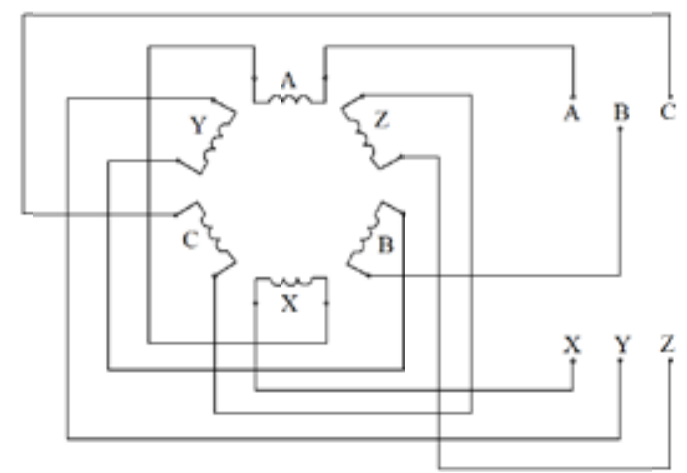

Рис. 2. Электрическая схема подключения катушек

Fig. 2. Electrical scheme of coils connection

Современные технологии проектирования позволяют проводить исследования электромагнитных, тепловых и гидродинамических процессов при помощи специализированных программных пакетов с достаточно высокой степенью достоверности. Использование методов математического моделирования при разработке электромагнитного перемешивателя дает возможность произвести предварительную оценку исследуемой конструкции установки, убедиться в правильности принятия технических решений.

Задача исследования - выявление картины электромагнитного поля в области слитка при кристаллизации, получение электромеханических характеристик, позволяющих дать предварительную оценку эффективности МГД-воздействия на жидкий металл.

\section{Описание математической модели}

В качестве исходных данных для расчета задают геометрические размеры элементов конструкции электромагнитного перемешивателя, кристаллизатора, физические свойства 
сред, электрические параметры питающей схемы. Для исследования трехмерной конечноэлементной модели используют программный пакет ANSYS Maxwell.

При построении математической модели приняты следующие допущения:

1. Решают переходную электромагнитную задачу с синусоидальными токами. Постановка задачи переходного типа выбрана в целях исследования электромеханических характеристик при различных скоростях вращения слитка.

2. Геометрию жидкой сердцевины определяют исходя из теплового расчета кристаллизующегося слитка.

3. Жидкая фаза слитка принята в качестве твердого тела.

4. Значения магнитной проницаемости магнитопровода задают согласно кривой намагничивания электротехнической стали Э11.

Система уравнений Максвелла, описывающих электромагнитные процессы в слитке, имеет вид:

$$
\begin{aligned}
& \nabla^{2} A=-\mu_{a} \delta, \\
& \nabla \delta=0, \\
& \delta=-\gamma \frac{A}{\partial t},
\end{aligned}
$$

где $A$ - векторный потенциал; $\delta$ - вектор плотности полного тока; $\mu_{a}$ - абсолютная магнитная проницаемость; $\nabla$ - оператор Лапласа; $\gamma$ - удельная электрическая проводимость, См/м.

Считая, что расчетную область окружает среда с идеальными магнитными свойствами $(\mu=\infty)$, на границах расчетной области будут выполняться условия:

- для касательной составляющей векторного потенциала

$$
\frac{\partial A_{\tau}}{\partial n}=0
$$

- для нормальной составляющей векторного потенциала

$$
A_{n}=0 \text {. }
$$

В качестве начальных условий принято, что в начальный момент времени скорость вращения слитка равна нулю.

\section{Результаты математического моделирования}

Проведено исследование электромагнитных процессов в области жидкой сердцевины слитка при различных параметрах питающей сети и расположении перемешивателя относительно слитка. Расчет производили при частотах тока $\mathrm{f}=10,20,30,40,50$ Гц. Выбор диапазона частот определяется исходя из требуемой глубины проникновения тока в жидкий металл, однако единых рекомендаций в теоретических работах до настоящего времени нет. Обычно глубину проникновения тока подбирают исходя из того, что она должна быть равна половине диаметра слитка или меньше. При перемешивании крупных слитков наиболее часто используется электромагнитное воздействие на пониженной частоте с целью интенсификации движения металла в глубинной части жидкой фазы. 
На рис. 3 представлена картина распределения мгновенных значений магнитной индукции в сечениях магнитопровода и слитка. Показатели магнитной индукции варьируют от $2,839 \cdot 10^{-2}$ до $174,1 \cdot 10^{-2}$ Тл в магнитопроводе и от $1,61 \cdot 10^{-4}$ до $146 \cdot 10^{-4}$ Тл в слитке. Насыщение электротехнической стали марки Э11 происходит при значениях магнитной индукции, превышающих 2 Тл. Следовательно, при установившемся режиме работы электромагнитного перемешивателя сталь магнитопровода полностью не насыщена, рассеяния магнитного потока вне сердечника не происходит.

Так как решаем переходный тип электромагнитной задачи, требуется определить время достижения установившегося режима работы. Анализируя зависимость электромагнитного момента от времени (рис. 4), видим, что амплитуда колебаний значительно снижается через 0,2 с после начала решения. Время достижения установившегося режима принимаем как $\mathrm{t}_{\mathrm{ycr}}=0,3 \mathrm{c}$. Временной шаг решения задачи $\mathrm{t}_{\mathrm{step}}=0,002 \mathrm{c}$, что достаточно для сходимости решения.

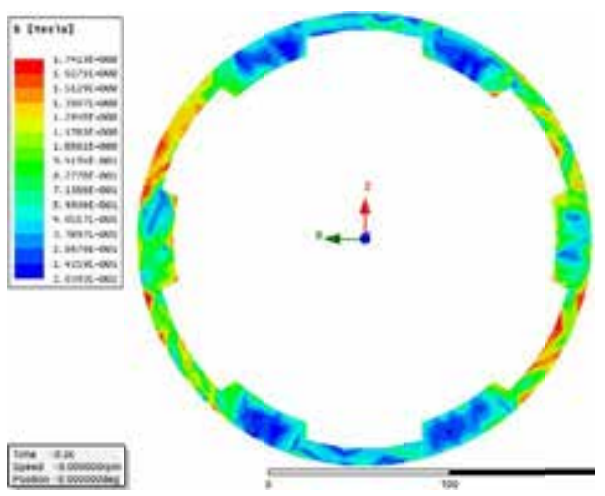

a

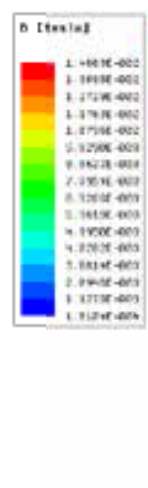

$\sigma$

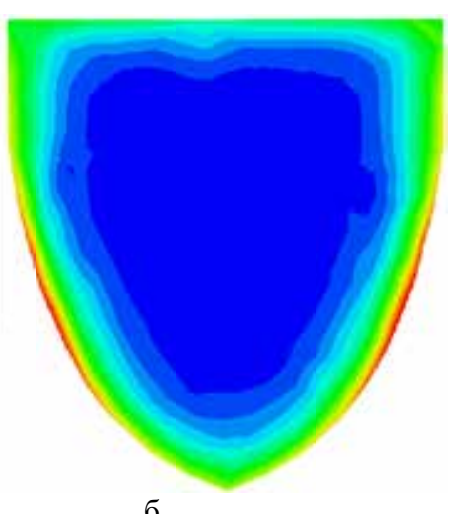

6

Рис. 3. Картина распределения мгновенных значений магнитной индукции: а-в сечении магнитопровода; б- в сечении слитка

Fig. 3. Distribution pattern of instantaneous values of magnetic induction: $a-$ in cross section of the magnetic core; $\sigma$ - in cross section of the ingot

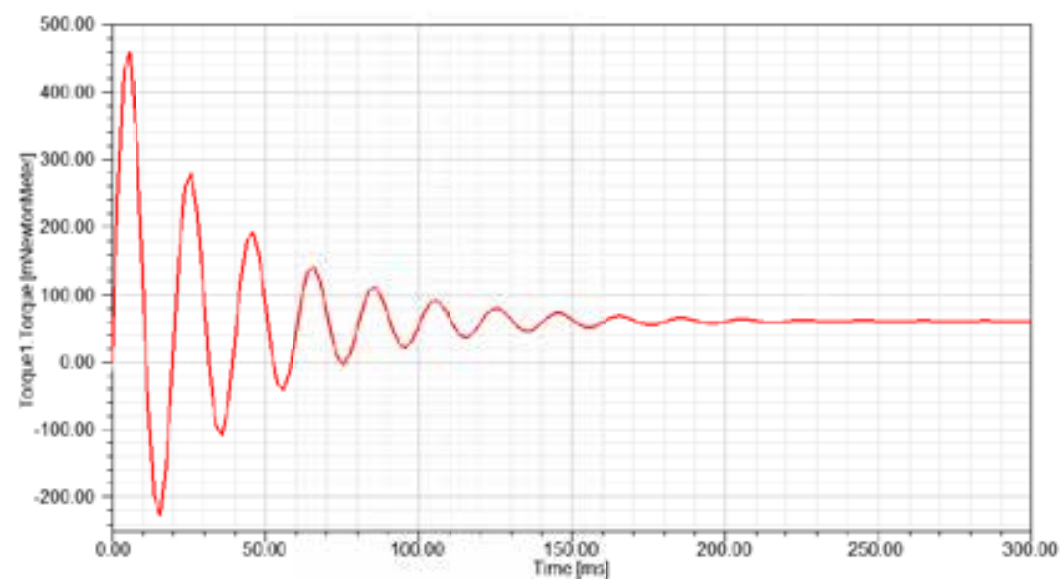

Рис. 4. Динамика колебаний амплитуды электромагнитного момента от времени решения задачи

Fig. 4. The dynamics of the amplitude of the electromagnetic moment on time task solution 


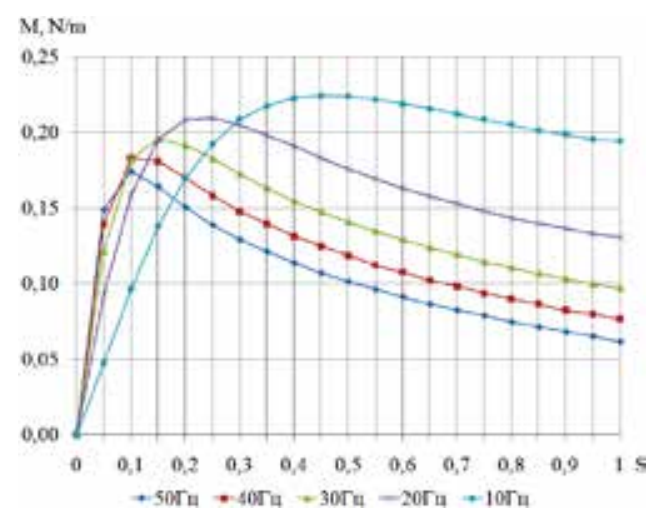

a

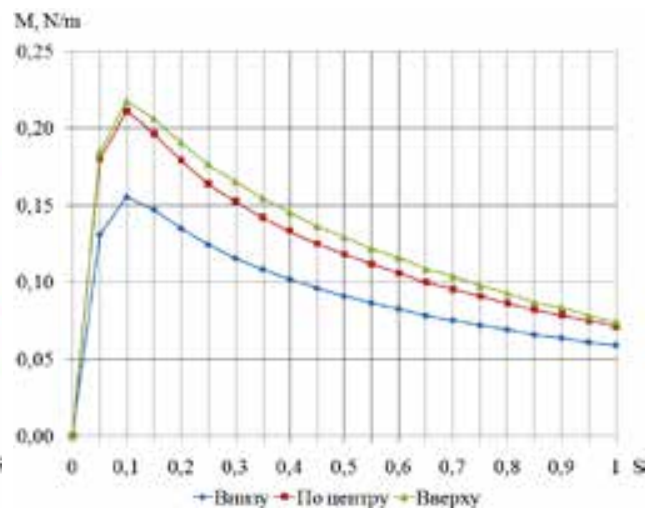

б

Рис. 5. Электромеханические характеристики перемешивателя: а - при различных частотах; б - при различном расположении относительно слитка

Fig. 5. Electromechanical characteristics of the stirrer: a - at different frequencies; $\sigma$ - at different locations relative to the ingot

Рассматривая электромагнитный перемешиватель в качестве электрической машины, необходимо оценить воздействие электромагнитных сил на слиток при различных значениях угловой скорости вращения, а также при трех вариантах расположения перемешивателя относительно жидкой сердцевины.

На рис. $5 a$ показаны электромеханические характеристики перемешивателя, полученные при заданных частотных режимах работы. Вращение области жидкой сердцевины осуществлялось с угловой скоростью в диапазоне требуемых значений.

Уравнение, определяющее среднюю величину вращающего момента в слитке:

$$
T=\int_{V o l} r \cdot(J \cdot B) d V o l
$$

где $r$ - вектор смещения от оси вращения; $J$ - плотность тока, А/м.

Исходя из полученных графиков следует, что наиболее высокие значения вращающих моментов наблюдаются при частотном диапазоне 10-20 Гц. Электромеханические характеристики имеют плавную форму за счет высоких пусковых моментов. При повышении частоты до 30-50 Гц глубина проникновения тока в слиток снижается, критический и пусковой моменты имеет самые низкие значения. Анализируя полученные электромеханические характеристики, можно сделать предварительный вывод, что перемешивание жидкого металла при частотном диапазоне 10-20 Гц является более предпочтительным за счет интенсификации воздействия электромагнитного поля на расплав. Окончательный выбор энергетических параметров установки следует осуществлять после проведения гидродинамических расчетов и экспериментальных исследований с использованием эвтектического сплава.

На рис. $5 б$ представлены электромеханические характеристики, полученные при различном расположении перемешивателя относительно слитка (рис. 6). Данный расчет проводили на частоте тока 50 Гц, без учета геометрии корпуса кристаллизатора, в целях возможности перемещения электромагнитного перемешивателя относительно области жидкой сердцевины.

$$
-957-
$$




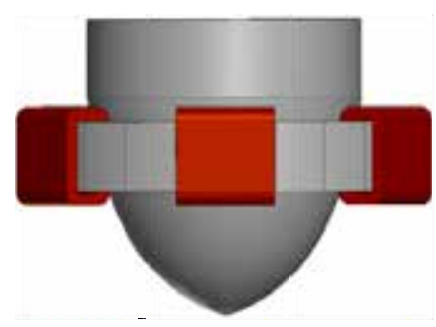

a

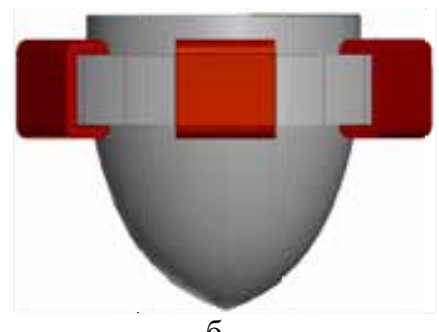

6

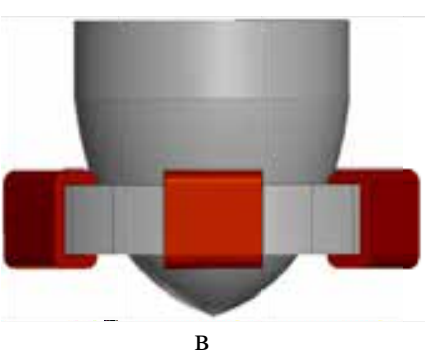

Рис. 6. Расположение перемешивателя относительно жидкой сердцевины: а - по центру; б - вверху; в внизу

Fig. 6. The location of the stirrer relative to the liquid heart: $\mathrm{a}-$ in the center; $\sigma-$ at the top; $\mathrm{B}-$ below

Наибольший электромагнитный момент наблюдается в случае расположения перемешивателя рядом с верхней частью слитка (рис. 6б), так как в данной области наименьший магнитный зазор между индуктором и жидкой сердцевиной. Минимальные значения электромагнитного момента получены при расположении, указанном на рис. 6в. При расположении перемешивателя относительно центра жидкой фазы (рис. $6 a$ ) электромеханическая характеристика незначительно отличается в меньшую сторону от характеристики, полученной при расположении перемешивателя в верхней части слитка (рис. 6б).

Для определения времени достижения номинальной скорости вращения жидкой сердцевины проведен расчет с момента неподвижного состояния слитка. В качестве начальных условий задана стартовая угловая скорость, равная нулю, а также расчетный момент инерции слитка.

Уравнение, определяющее угловую скорость вращения слитка:

$$
n=n_{0}(1-S),
$$

где $\mathrm{n}_{0}$ - частота вращения магнитного поля, об/мин; $\mathrm{S}$ - скольжение.

Уравнение, определяющее расчетный момент инерции слитка:

$$
J=\frac{m R_{c p}^{2}}{2},
$$

где $\mathrm{m}$ - масса слитка; $R_{c p}$ - средний радиус слитка.

На рис. $7 a$ представлены электромеханические характеристики угловой скорости от времени при различных частотах. Достижение номинальной скорости вращения ротора при частоте 50 Гц происходит через 96 с. При частоте 10 Гц время достижения номинальной скорости сокращается до 15 с. На рис. 76 отражены электромеханические характеристики электромагнитного момента от времени. Достижение максимальных значений электромагнитного момента при частоте 50 Гц происходит через 85 с. При частоте 10 Гц время достижения максимального электромагнитного момента составляет $8 \mathrm{c}$.

Для обеспечения требуемого режима литья следует использовать периодические включения и отключения установки, чтобы скорость вращения слитка не достигала синхронной. По результатам проведенных исследований [5-7] выяснили, что наиболее часто используемый диапазон скоростей при электромагнитном перемешивании жидкой сердцевины варьируется

$$
-958-
$$




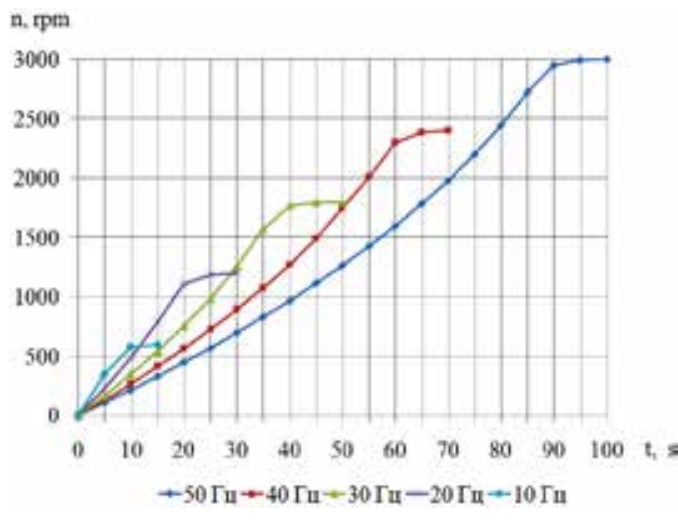

a

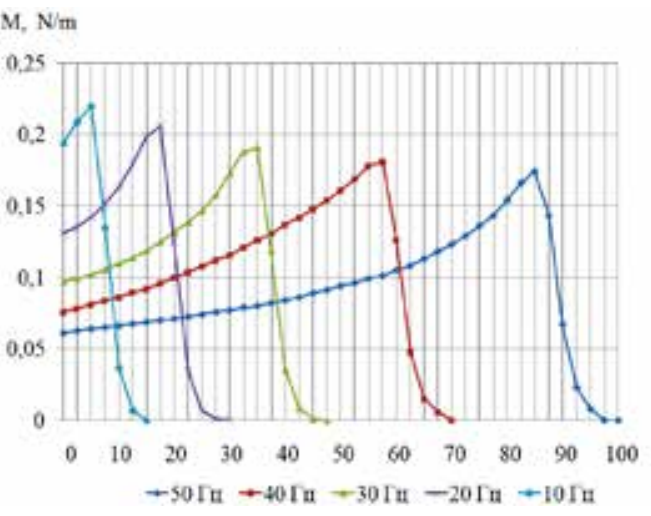

6

Рис. 7. Электромеханические характеристики: а - угловой скорости от времени; б - электромагнитного момента от времени

Fig. 7. Electromechanical characteristics: $a$ - angular velocity from time; $\sigma$ - electromagnetic moment from time

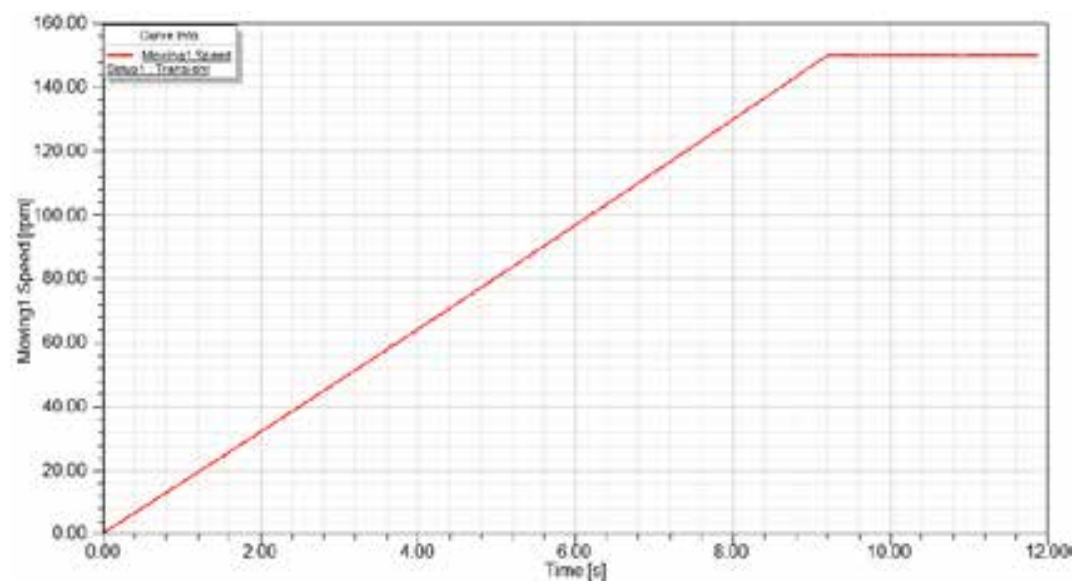

Рис. 8. Динамика изменения угловой скорости слитка от времени расчета

Fig. 8. The dynamics of changes in the angular velocity of the ingot from the calculation time

от 0,2 до 0,3 м/с. В данном случае следует, что угловая скорость вращения в процессе перемешивания не должна превышать 150 об/мин. При более высоких скоростях возможно размытие раздела твердой и жидкой фаз, повреждение поверхностной окисной пленки. Процесс подачи питания на катушки осуществляется при помощи использования условных выражений, регулирующих величину тока, в зависимости от угловой скорости слитка. Время достижения требуемой угловой скорости вращения слитка составило 9,2 с при частоте тока 50 Гц (рис. 8). В течение последующего времени расчета скорость вращения не превышает 150 об/мин.

\section{Результаты физического моделирования}

Достоверность математического моделирования подтверждена в ходе сравнения расчетного распределения действующих значений горизонтальной составляющей магнитной индукции 
Таблица 1. Результаты измерения горизонтальной составляющей магнитной индукции при 10 и 50 Гц Table 1. The results of measuring the horizontal component of magnetic induction at $10 \mathrm{and} 50 \mathrm{~Hz}$

\begin{tabular}{|c|c|c|c|c|}
\hline $\begin{array}{c}\text { № контрольной } \\
\text { точки }\end{array}$ & $\begin{array}{c}\text { В, Тл при } \\
\mathrm{f}=10 \text { Гц, изм. }\end{array}$ & $\begin{array}{c}\text { В, Тл при } \\
\mathrm{f}=10 \text { Гц, расч. }\end{array}$ & $\begin{array}{c}\text { В, Тл при } \\
\mathrm{f}=50 \text { Гц, изм. }\end{array}$ & $\begin{array}{c}\text { В, Тл при } \\
\mathrm{f}=50 \text { Гц, расч. }\end{array}$ \\
\hline 1 & 0,0046 & 0,0042 & 0,0018 & 0,0021 \\
\hline 2 & 0,0042 & 0,0036 & 0,0011 & 0,0014 \\
\hline 3 & 0,0038 & 0,0033 & 0,00097 & 0,00108 \\
\hline 4 & 0,0079 & 0,0091 & 0,002 & 0,0023 \\
\hline 5 & 0,0044 & 0,0039 & 0,0013 & 0,00145 \\
\hline 6 & 0,0041 & 0,0037 & 0,00109 & 0,00127 \\
\hline
\end{tabular}

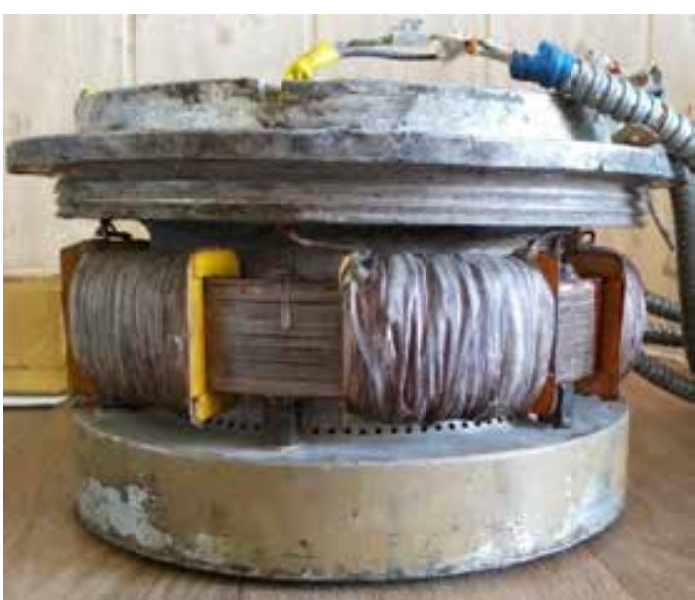

a

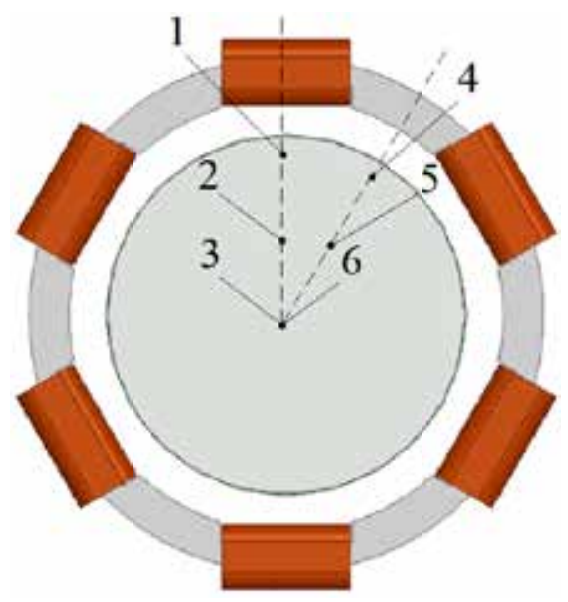

6

Рис. 9. Общий вид физической модели электромагнитного перемешивателя (а) и схема расположения контрольных измерительных точек (б)

Fig. 9. General view of the physical model of the electromagnetic stirrer (a) and layout of control measuring points (б)

и экспериментальных данных, полученных на физической модели электромагнитного перемешивателя (рис. 9a). Оценка результатов электромагнитного расчета проведена при помощи стенда для измерения действующих значений магнитной индукции поля, основанного на датчике Холла. Используя контрольные измерительные точки аналогично их расположению в математической модели (рис. 9б), проведены замеры горизонтальной составляющей магнитной индукции по оси катушек и магнитопровода. Измерения осуществлены при частотах $\mathrm{f}=10$, 50 Гц. Относительное расхождение измеренных и расчетных результатов не превысило 15 \%.

\section{Заключение}

Разработанная математическая модель позволяет находить распределение электромагнитного поля перемешивателя, получать электромеханические характеристики при различных параметрах питающей сети. Анализируя найденные результаты, делаем вывод, что электромагнитное воздействие на слиток наиболее эффективно при частотном диапазоне 10-20 Гц.

$$
-960-
$$


Использование цикличного режима управления питанием установки является необходимым условием для осуществления процесса перемешивания жидкой сердцевины слитка.

Исследование выполнено при финансовой поддержке РФФИ и Правительство Красноярского края в рамках научного проекта №18-48-242013 «Исследование влияния пространственных и частотно-временных распределений электромагнитных сил в расплаве на характер протекания МГД-процессов в жидком металле».

\section{Список литературы}

[1] Напалков В.И., Черепок Г.В., Махов С.В., Черновол Ю.М. Непрерывное литье алюминиевых сплавов. М.: Интермет Инжиниринг, 2005. 512 с. [Napalkov V.I., Cherepok G.V., Mahov S.V., Chernovol Y.M. The continuous casting of aluminum alloys. Moscow, Intermet Engineering, 2005, 512 p. (in Russian)]

[2] Ефимов В.А., Эльдарханов А.С. Технологии современной металлургии. М.: Новые технологии, 2004. 794 с. [Efimov V.A., El'darhanov A.S. The Technologies of the Modern metallurgy. Moscow, New technologies, 2004, 794 p. (in Russian)]

[3] Гецелев 3.Н., Балахонцев Г.А., Квасов Ф.И., Черепок Г.В., Варга И.И., Мартынов Г.И. Непрерывное литье в электромагнитный кристаллизатор. М.: Металлургия, 1983. 152 с. [Getselev Z.N., Balakhontsev G.A., Kvasov F.I., Cherepok G.V., Varga I.I., Martynov G.I. The continuous casting in an electromagnetic crystallizer. Moscow, Metallurgy, 1983, 152 p. (in Russian)]

[4] Ливанов В.А., Габидуллин Р.М., Шипилов В.С. Непрерывное литье алюминиевых сплавов. М.: Металлургия, 1977. 168 c. [Livanov V.A., Gabidullin R.M., Shipilov V.S. The continuous casting of aluminum alloys. Moscow, Metallurgy, 1977, 168 p. (in Russian)]

[5] Li Wu, Hui-jun Kang, Zong-ning Chen, Ning Liu, Tong-min Wang. Horizontal continuous casting process under electromagnetic field forpreparing AA3003/AA4045 clad composite hollow billets. Trans. Nonferrous Met. Soc. China, 2015, 25, 2675-2685.

[6] Христинич Р.М., Христинич А.Р., Христинич Е.В. Энергетические характеристики индукционной машины с жидкометаллическим рабочим телом. Вестник Красноярского государственного аграрного университета, 2013, 9, 218-223 [Hristinich R.M., Hristinich A.R., Hristinich E.V. Power characteristics of the induction machine with a liquid working rotor, Bulletin of the Krasnoyarsk state agricultural university, 2013, 9, 218-223 (in Russian)]

[7] Cramer A., Eckert S., Galindo V., Gerbeth G., Willers B., Witke W. Liquid metal model experiments on casting and solidification processes. Journal of Materials science, 2004, 39, $7285-$ 7294. 